\section{Psicología IBEROAMERICANA}

\section{Psicología Iberoamericana}

\section{ISSN: 1405-0943}

psicología.iberoamericana@uia.mx

Universidad Iberoamericana, Ciudad de México

México

Gutiérrez Garay, Oskar

Caracterización y diagnóstico de personas en proceso de retiro laboral de la Secretaría Distrital de Salud de Bogotá

Psicología Iberoamericana, vol. 22, núm. 1, enero-junio, 2014, pp. 34-44

Universidad Iberoamericana, Ciudad de México

Distrito Federal, México

Disponible en: http://www.redalyc.org/articulo.oa?id=133944229005

- Cómo citar el artículo

Número completo

- Más información del artículo

- Página de la revista en redalyc.org

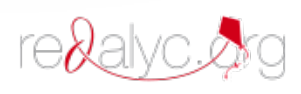

Sistema de Información Científica

Red de Revistas Científicas de América Latina, el Caribe, España y Portugal Proyecto académico sin fines de lucro, desarrollado bajo la iniciativa de acceso abierto 


\title{
Caracterización y diagnóstico de personas en proceso de retiro laboral de la Secretaría Distrital de Salud de Bogotá
}

\author{
Characterization and diagnosis of people in the process \\ of retiring from Bogota's Public Health Department
}

\section{RESUMEN}

El siguiente artículo aborda la investigación mixta que hizo el Observatorio para personas en proceso de retiro laboral de la Universidad Manuela Beltrán, para caracterizar y diagnosticar en las áreas de salud, familia, finanzas, comunitaria y personal a un grupo de 131 adultos mayores que trabajan para la Secretaría Distrital de Salud de Bogotá y que están en proceso de retiro laboral por derecho a pensión.

Palabras clave: retiro laboral, jubilación, área personal, familiar, comunitaria, salud.

\section{ABSTRACT}

This article studies the mixed research by the Observatory for people in the process of retiring of the Manuela Beltrán University, to characterize and diagnose the health, family, financial, community and personal issues of a group of 131 senior adults working for Bogotás Public Health Department and who are currently in the process of retiring, having qualified for a pension.

Keywords: retirement, family, community, personal, health.

\footnotetext{
${ }^{1}$ Recibido: 4 de agosto de 2013 - Aceptado: 7 de abril de 2014.

Docente en la Universidad Manuela Beltrán de Bogotá. Candidato al Doctorado en Pensamiento Complejo de la Multiversidad Mundo Real. Para correspondencia escribir a: oskargutierrez@gmail.com o Av. Circunvalar \# 60-00 Bogotá, Colombia. Tel. (57)3162251744.
} 


\section{INTRODUCCIÓN}

La Secretaría Distrital de Salud (SDs) es la "entidad rectora de salud en Bogotá (...) responsable de garantizar el ejercicio efectivo del derecho a la salud de toda la población, a través de la implementación de un modelo de atención integral, equitativo, universal, participativo, centrado en el ser humano, la responsabilidad social y la sostenibilidad ambiental" (sDs, 2013).

Consciente y consecuente de su misión institucional, la SDs firmó un convenio con la Universidad Manuela Beltrán (UMB) para desarrollar la fase 1 del Observatorio para personas en proceso de retiro laboral, con los funcionarios de la sDs que están a punto de recibir su pensión. Para esto, se estableció un proceso que tuvo tres momentos: 1) construcción de instrumentos cuantitativos y cualitativos para conocer el estado actual y las necesidades de la población, que brindan una caracterización demográfica cuantitativa y unas categorías de análisis cualitativas de la información recolectada; 2) aplicación de la encuesta y la entrevista estructurada, que permitieron la recolección directa de la información de la población; 3) análisis de resultados, en los cuales puede observarse la caracterización sociodemográfica y el análisis desde las categorías: a) salud, b) personal, c) familiar, d) comunitaria, y e) financiera.

\section{CONSTRUCCIÓN DE INSTRUMENTOS}

El equipo de investigadores construyó dos instrumentos de recolección de información, validados por jueces, con características cuantitativas y cualitativas.

La encuesta, con el objetivo de recolectar información sociodemográfica e información del área de salud de la población, estuvo compuesta por varios ítems que consideraron: edad, género, estrato, escolaridad, actividades extralaborales y antecedentes de salud personales y familiares.

Con el fin de recolectar las narrativas de los prepensionados alrededor de las áreas familiar, personal, salud, comunitaria y financiera, se diseñó una entrevista estructurada con ítems que permitieran ampliar la información en cada una de las categorías planteadas.

Se llevaron a cabo 88 entrevistas correspondientes a $63 \%$ del total de la población y 131 encuestas que corresponden a $100 \%$ de la población. La entrevista fue opcional, a diferencia de la encuesta que fue obligatoria, según la orden dada directamente por el área de Gestión Humana de la sDs y avalada por el secretario general. Este proceso contó con la participación de 16 auxiliares de investigación y de tres docentes investigadores.

\section{ANÁLISIS DE RESULTADOS}

El análisis de los datos cuantitativos que arrojó la encuesta puede observarse en el siguiente apartado. Asimismo, las entrevistas aplicadas arrojaron narrativas que fueron transcritas para poder elaborar el análisis categorial.

\section{Análisis cuantitativo \\ Análisis sociodemográfico}

Otra situación que se tuvo en cuenta para el conocimiento de las características de la población de prepensionados de la sDs fueron los resultados obtenidos en la encuesta que se aplicó a la par de las entrevistas. Dicha encuesta reúne información relevante sobre aspectos sociodemográficos generales de la población, e indaga con mayor profundidad sobre los hábitos de actividad física, trabajo comunitario, emprendimiento $y$, en especial, acerca de aspectos de salud actual y perfil de riesgos de la población.

La primera característica que se consideró para describir a la población fue la condición de género, en la que se determinó que la muestra está compuesta por $59.4 \%$ de hombres y $48.6 \%$ de mujeres, ambos entre los 53 a los 66 años de edad. Otro aspecto que identifica a la población es que en en más de la mitad de los casos los prepensionados están casados, y en menor proporción, inferior a $15 \%$, se encuentran en otros estados civiles: soltero, unión libre, divorciado y viudo.

En cuanto a la escolaridad de los prepensionados, se pudo determinar que la mayoría de las personas que aspiran a jubilarse, predominantemente han culminado una especialización, mientras que un valor cercano a $20 \%$ terminó estudios de maestría y, en menor proporción, las personas han alcanzado estudios técnicos, tecnológicos, universitarios o de bachillerato, mientras que en una baja proporción se encuentran aquellos que sólo cursaron estudios de educación básica primaria.

Otro aspecto que se tomó en cuenta dentro de la caracterización de los pre-pensionados de la sDs fue 
su distribución geográfica en la ciudad, se pudo conocer que la mayoría de las personas residen en Suba en 24\% de los casos, seguido de las localidades Engativá, Kennedy y Usaquén, en menor proporción y otras localidades con menos frecuencia; en este mismo sentido, la mayoría de los prepensionados habitan en viviendas clasificadas dentro de zonas estrato 3 en $47 \%$ de los casos; en $33 \%$ en zonas estrato 4 , y en menor proporción, en viviendas de otros estratos socioeconómicos.

Considerando que una de las situaciones que suelen preocupar más a los prepensionados es el aseguramiento de su manutención económica posterior a la jubilación, la información sobre la estructura económica actual de estas familias, resulta de interés para la caracterización de la población. Al respecto, se pudo determinar que en mucho más de la mitad de los casos, los prepensionados tienen vivienda propia. Adicionalmente, pudo determinarse que, en general, las familias de los prepensionados son nucleares, en las que en promedio existen entre dos y tres personas que dependen económicamente del sujeto.

En cuanto a las actividades emprendedoras de los prepensionados, se logró establecer que la mayoría de estas personas no ha intentado en la actualidad el desarrollo de alguna actividad emprendedora, ya que cerca de $60 \%$ de los encuestados afirmó no haber iniciado ningún tipo de emprendimiento. Resulta interesante mencionar que entre aquellos que iniciaron algunas actividades económicas independientes, $90 \%$ adelantó sólo la planeación de su emprendimiento, la formación de empresas en servicios y asesorías y gestión de proyectos, principalmente, y sólo en algunos casos estos proyectos ya se encuentran en ejecución y se trata de ventas de fin de semana y de fin de año.

\section{Área salud}

En cuanto al área de salud, se realizó una caracterización de los aspectos de salud de la población encuestada, en primera medida, al estimar que las enfermedades que suelen desarrollarse en el adulto mayor tienen un importante componente genético en un alto porcentaje. Debido a ello, se consideró la evaluación de los antecedentes paternos y maternos en cada uno de estos cuadros clínicos. Se logró determinar que en una baja proporción las personas presentan riesgo genético de padecer alguno de dichos cuadros, ya que se pudo demostrar que sólo un bajo porcentaje de los encuestados ha reportado la existencia de estas enfermedades en sus padres. Conviene mencionar que el mayor número de casos corresponde a antecedentes médicos relacionados con enfermedad vascular, entre los que destacan la hipertensión arterial, infartos y, en general, formas de enfermedad cardiaca.

Asimismo, se realizó una evaluación de antecedentes médicos del grupo de prepensionados. Al respecto, se determinó que en cuanto a la existencia de antecedentes quirúrgicos, $60 \%$ de los encuestados ha pasado por algún tipo de cirugía, entre las que predominan la apendicectomía, la extracción de amígdalas e intervenciones relacionadas con la ortopedia, como la atención de fracturas y tratamiento para la enfermedad de ligamentos y articular; en menor medida, se pudo establecer la existencia de intervenciones para la extracción de cálculos biliares y renales, así como el tratamiento para otras enfermedades del riñón. En menor proporción, y de manera específica para la población femenina, conviene mencionar las cirugías ginecoobstétricas, entre las que resaltan los partos por cesárea y la histerectomía.

En cuanto a los antecedentes hospitalarios, se obtuvo el dato de que $80 \%$ de los encuestados ha tenido alguna forma de hospitalización; sin embargo, en una alta proporción, estos casos concuerdan con los antecedentes quirúrgicos; es decir, que la hospitalización va asociada a la intervención quirúrgica, sólo en $12 \%$ de los casos estudiados, las hospitalizaciones estuvieron asociadas a condiciones médicas diferentes, como úlcera gástrica y otras enfermedades gastrointestinales, por menos de 10 días, al igual que en casos de resultantes de enfermedad vascular, como la trombosis venosa.

Respecto a los antecedentes farmacológicos, se pudo determinar que en la actualidad, sólo $25 \%$ de los encuestados consume algún tipo de medicamento de manera continua e ininterrumpida; predominan los medicamentos para el tratamiento de la hipertensión arterial y los reguladores hormonales para la tiroides y paratiroides, y en menor proporción, para el tratamiento de la hipoglicemia y diabetes.

Finalmente, en lo relativo a antecedentes patológicos de los prepensionados de la SDS, se estudiaron al detalle por sistemas funcionales y etiologías. $\mathrm{Al}$ respec- 
to, se pudo determinar que en cuanto a formas de enfermedad se destacan los padecimientos oculares, que ocurren en $40 \%$ de los sujetos encuestados, entre quienes la miopía se presenta como cuadro predominante, seguida por la miopía combinada con astigmatismo.

Otro antecedente interesante para la descripción de las características patológicas de los encuestados, se refiere a los padecimientos de cuadros osteo-musculares. Se pudo establecer que aproximadamente $20 \%$ de los encuestados reporta algún tipo de enfermedad relacionada con este sistema, en especial, reportan la artrosis y mialgia como la queja predominante, seguida de los síntomas propios del síndrome del túnel del carpo. Otras formas de padecimientos no superan 3\% del total de casos reportados.

En cuanto a enfermedades relacionadas con el sistema gastrointestinal, se determinó que $20 \%$ de los encuestados manifiestan tener algún tipo de alteración en este sistema; la mayoría de estos casos constituye una forma de gastritis y, en menor proporción, colon irritable.

Respecto a los antecedentes cardiovasculares de los encuestados, se pudo determinar que $11 \%$ de los prepensionados participantes en el estudio reportan algún tipo de alteración cardiovascular; es significativa la presencia de hipertensión arterial en relación con otras formas de enfermedad de este sistema.

Acerca de los resultados estimados para el registro de los antecedentes patológicos de los encuestados en otras áreas, se pudo conocer que los reportes de enfermedad no representan proporciones significativas, ya que el número de personas que enfrentan algún tipo de padecimiento en ninguno de los casos supera $8 \%$ del total de encuestados. Por ejemplo, para el caso de los antecedentes de enfermedad dermatológica, se pudo determinar la existencia de 11 casos correspondientes a $7.5 \%$ del total de de la muestra, quienes padecen principalmente de formas de alergia o dermatitis (nueve casos) y dos casos de vitíligo. Otra característica que se tuvo en cuenta fue la consideración de aquellos encuestados que reportan enfermedad pulmonar. Se registró que entre estas personas prepensionadas, $5 \%$ tiene algún tipo de padecimiento relacionado con este sistema, en específico, se conocieron ocho casos de bronquitis, tres de los cuales se complejizaron a neumonía y tres casos de asma.
Finalmente, en antecedentes neurológicos se registraron dos casos, equivalentes a $1.7 \%$. El primero reporta padecer un cuadro de migraña, mientras que otro menciona un aneurisma por el que recibió tratamiento quirúrgico; en este mismo sentido, en cuanto a antecedentes psiquiátricos y psicológicos, se pudo conocer la existencia de dos casos de consulta por alteraciones de ansiedad y un caso de depresión que tuvo tratamiento farmacológico y hospitalización.

\section{Análisis cualitativo \\ Área familiar}

Según Cuartas (1999), el retiro laboral implica no sólo para la persona a punto de pensionarse una serie de cambios en su vida, también se ve involucrada su familia debido a que, posiblemente, se va a empezar a tener una disponibilidad de tiempo absoluta. Es aquí donde se requiere conocer las relaciones que se tienen con los miembros de la familia, qué tanto han hablado del tema, si se dispone o no de actividades, planes o proyectos y si desean o cuentan con alguien de su familia para desarrollarlas.

Es evidente que los sujetos se enfrentan a la elaboración de un proceso de duelo (cierre de una etapa) donde las rutinas, patrones y relaciones dan un giro importante, que hacen que el sujeto se enfrente a nuevos retos y dinámicas. La familia cobra una función determinante, ya que las dinámicas relacionales también cambian en las personas que constituyen la red de apoyo, que ahora tienen que aprender, acomodarse y facilitar el nuevo papel.

Antes de cumplir los requisitos legales y tener conocimiento de que se aproxima esta etapa, es importante iniciar una preparación; esto ayuda a la toma de conciencia, así como a conocer cuáles son las etapas por las que se va a pasar. Hay varias fases en este proceso de jubilación (Giró, 2009).

Primero, se presenta la fase de prejubilación, donde la persona va tomando conciencia del significado de la jubilación, se pregunta cómo será ésta; tiene expectativas, para luego entrar en la fase de jubilación como tal, en la que hay cuatro tipos de vivencias: la primera se denomina luna de miel, en la que las personas intentan hacer todo lo que desearon hacer y no pudieron en los años anteriores; la segunda es la fase de rutina, que se caracteriza porque se tienen actividades y grupos 
estables, con los que únicamente hay que cambiar el tiempo dedicado a estas actividades; la tercera es la fase de rélax y descanso, que se caracteriza por una reducción temporal de la actividad y, finalmente, está la fase de desencanto y depresión, que es cuando la persona jubilada comprueba que sus expectativas, ideas y fantasías sobre la jubilación no se cumplen, para seguir con una parte de reorientación, en la cual se pasa por una reevaluación y elaboración de propósitos y proyectos nuevos acordes con la realidad del jubilado, según las características de cada persona.

Al pasar por estas fases se llega a desarrollar un estilo de vida rutinario posterior a la jubilación, se generan medios para afrontar los cambios producidos como consecuencia de la jubilación, "aquellas personas con rutinas satisfactorias de jubilado son conscientes de sus capacidades y limitaciones, conocen lo que se espera de ellos y tienen su propio rol o papel definido, lo cual se ajusta a la fase final del proceso de jubilación" (Giró, 2009, p. 52).

Estas fases son claves en un proceso de preparación, pues los temas relacionados deben ser acordes a las etapas o fases por las que se va pasando, así, al tener conocimiento de ellas, se van poniendo en práctica las habilidades para afrontarlas de la mejor forma y obtener buenos resultados y una mejor planificación. "La preparación para la jubilación proporciona una mejor aceptación frente a esa nueva etapa de la vida, permite un mejor desenvolvimiento personal, social y familiar" (Cuartas, 1999, p. 13).

Es cierto que en este primer momento estarían en la primera fase de las descritas por Giró (2009); sin embargo, según las historias de vida, el contexto de cada persona y las situaciones, pueden devenir en fases distintas con intensidades y cualidades muy particulares.

En este momento, al preguntarles a los entrevistados por una proyección a cinco años, las historias aunque particulares, apuntan en muchas ocasiones a lo mismo, en especial al papel de su familia y cómo el tiempo que van a pasar con ellos es determinante para los objetivos y su calidad de vida. Por ejemplo, esto es algo que manifiesta una de las participantes de las entrevistas: “¿Cómo me veo en cinco años? Abuela. Ya soy abuela, entonces me veré más abuela, entonces vo a pasarla muy sabroso porque amo a mi nieta, la amo terriblemente y más que se parece a mí (risas)”.
La familia nuclear es importante pero la familia extendida, quizá lo sea más. Los nietos, bisnietos, el reencuentro y el reconocimiento de la relación de pareja indudablemente propenderán por el bienestar en todo el sistema familiar que se busca. Los roles cambian y la relación entre las personas también cambia.

Después de esta primera fase se presenta el desarrollo de un estilo de vida rutinario posterior a la jubilación, donde se reacomodan las rutinas y se generan medios para afrontar los cambios producidos como consecuencia de la jubilación. Aquellas personas con una rutina satisfactoria de jubilado son conscientes de sus capacidades y limitaciones, conocen lo que se espera de ellos y tienen asumido su propio rol o papel. Para algunas personas, el papel del jubilado es irrelevante debido a que vuelven al trabajo o debido a una enfermedad o una incapacidad.

Estas fases son importantes en el proceso de preparación, pues los temas relacionados con ésta deben ser acordes a las etapas o fases por las que se va pasando y al tener conocimiento de ellas, se van colocando en práctica las habilidades para afrontarlas. Como lo describen Potocnik, Tordera y Peiró (2008), la jubilación es la pérdida de la actividad laboral contratada. Al presentarse una pérdida se presenta un duelo, en este caso, un duelo laboral, el cual debe ser manejado con una adecuada preparación que permita afrontar los cambios con estrategias de reestructuración, transformación y modificación de hábitos y rutinas.

No sólo se presenta pérdida de la actividad laboral, además se presentan pérdidas que concuerdan con la etapa, por ejemplo, pérdidas familiares o de fuentes proveedoras de afecto, asociadas con sentimientos de soledad y desamparo; se pueden presentar pérdidas de objetos materiales, o sentimientos de pérdida de estatus, reconocimiento e identidad social (Placeres, Lázaro \& Delgado, 2011).

Dentro de las preocupaciones que más afligen a los prejubilados, se encuentra el área financiera, debido a que el retiro laboral también puede implicar una modificación en la economía de la familia. Dado lo anterior se puede observar que hay varias familias que son dependientes económicamente del prejubilado, lo que en el momento del retiro laboral puede desestabilizarlas, y ocasionar problemas de salud y presiones psicosociales en el individuo, puesto que hay una pér- 
dida de ingresos económicos que dificulta el mantenimiento de los niveles de vida de los familiares.

"Algunas investigaciones han señalado que uno de los objetivos de los trabajadores en los procesos de prejubilación es obtener una indemnización lo más elevada posible para cubrir el periodo de retiro temprano hasta la edad de jubilación" (Fernández, Crego \& Alcover, 2008, p. 435). Algunas personas presentan dificultades económicas al no contar con los recursos adecuados, debido a que deben estar sometidos a una pensión que seguramente no alcanza para suplir sus necesidades inmediatas. Algunos entrevistados manifiestan su deseo de seguir laborando o dedicarse a algo que mezcle una actividad placentera con una fuente de ingresos.

Como contingencia a esto, y de acuerdo con De Freitas y Vaughan (2008), es importante que durante un tiempo previo a la jubilación se establezcan foros permanentes de discusión y actividades de capacitación sobre el nivel de vida que los trabajadores quieren tener en el momento del retiro laboral, con el fin de hacer que tomen conciencia de los riesgos y animarlos para que se preparen y piensen en su bienestar personal y familiar a futuro. Acá el acompañamiento de la SDs es vital para evitar situaciones como la que se evidencia en la entrevista de una de las participantes:

No sé, quisiera hacer algo con un ingreso que me genere una entrada que empiece a desarrollar, pero la verdad no he podido encontrar, no se me ha ocurrido, te cuento, no se me ha ocurrido, he estado tratando de buscar desde diferentes campos a ver qué se podría hacer pero no me encuentro la respuesta, porque empezar a hacer empresa a estas alturas de la vida, no le veo...

\section{Área financiera}

Los entrevistados mencionan que tienen ahorros con los que pueden contar en caso de presentarse cualquier situación, estos ahorros también los han proyectado para adquirir bienes o invertir en un negocio que les genere un ingreso adicional, ya que refieren que una mesada pensional no es suficiente para sostenerse económicamente, como lo manifiesta uno de los entrevistados:
... probablemente te pueda afectar, por eso yo te digo que a largo plazo, a mediano, mediano-largo plazo yo no me veo dependiendo de mi pensión únicamente, porque pues como están las condiciones económicas del país, pues uno dice "bueno, de aquí a cinco años que yo me pensione", y haciendo uno los cálculos y uno se pone a hacer cálculos factoriales de eso, pues uno terminaría pensionándose con dos, tres millones de pesos de aquí a cinco años, pues eso no te da ninguna garantía.

Ese aspecto familiar está directamente ligado al aspecto financiero, la mayoría no entiende uno sin el otro, ya que el aspecto financiero beneficiará o afectará directa y proporcionalmente a su familia.

Pese a que se tiene una cultura del ahorro, los entrevistados evidencian no haber proyectado una situación que los llegase a dejar sin solvencia económica. Este aspecto incide de manera importante en la vida del pensionado, ya que sus aspiraciones se basan en tener una estabilidad económica en su vejez, sin depender ni ser una carga para nadie, es la satisfacción y la recompensa a todo el trabajado realizado por años.

En esta parte se articulan las demás áreas, puesto que el dinero es un condicionante para que haya un bienestar general, como lo manifiesta uno de los entrevistados: "Si me he pensionado espero estar haciendo docencia o seguramente me quiero trasladar a otro país con mi familia, eso es lo que he pensado".

En el proceso, se puede presentar un cambio de roles que puede generar una nueva estructura familiar, en la que es vital conocer qué tanto dialogan, si las decisiones de los familiares se tienen en cuenta en el momento de tomar una decisión.

Eguiluz (2007) identifica que una pareja en su vejez y jubilación, sin la presencia de sus hijos, puede volver a retomar su relación conyugal, pero esto es una tarea un poco más compleja, ya que muchos se han dedicado por años a sus hijos, o a su trabajo sin atenderse a sí mismos. Ambos deben enfrentarse a la jubilación, esto provoca cambios en todas sus rutinas, horarios, actividades y economía familiar, asunto que tiende a generar crisis.

Lo anterior se da inicialmente por parte de las mujeres, al no saber qué hacer con sus esposos ahora que están todo el día en casa; y para los esposos, que han 
trabajado toda su vida, les resulta difícil estar todo el día en el hogar y no saben qué hacer en su tiempo libre. La mayoría de estas personas no saben cuáles son sus aficiones, y por falta de información no conocen personas en su misma condición con las que puedan interactuar y compartir.

Eguiluz (2007) afirma que en los casos de enfermedades degenerativas o crónicas, sean físicas o psíquicas, uno de los dos integrantes cumple el rol de cuidador, lo cual no resulta fácil; o los papeles se invierten, siendo los hijos quienes asumen el papel de cuidador, lo cual puede generar una crisis.

Cuando los entrevistados hablan de sí mismos, les es imposible dejar de hablar de sus logros y de los miembros de su familia, especialmente de sus hijos. Algunos se refieren a ellos dentro de sus planes de proyecto de vida, como en el caso de uno de los entrevistados, quien ante la pregunta de si había algún proyecto que no hubiera podido realizar, respondió: "De pronto, el compartir más con mis hijos".

El bienestar familiar para las personas entrevistadas se resume en tener una buena relación entre sus miembros, así como en brindar las condiciones necesarias para garantizar una estabilidad y un futuro promisorio. $\mathrm{Al}$ respecto, una de las entrevistadas expresa: "algo muy bonito para mí, situaciones importantes, fue haber logrado tener una estabilidad laboral. Otra parte importante para mí [fue] el nacimiento de mis hijos, otra parte importante para mi vida es haber tenido dos experiencias matrimoniales".

Siendo el tema familiar el aspecto que más puntúa como significativo, se parte del hecho de que la familia se convierte en una red de apoyo primordial que permite la autorrealización en aspectos relevantes de la vida.

Tomando como referente lo anterior, se podría decir que dentro de una familia debe haber una funcionalidad donde todos participen y aporten, generando así un equilibrio; no obstante, en las algunas personas indagadas a través de la entrevista, existe la figura familiar, pero en otras no. O es muy dinámica. Para ejemplificar lo anterior, un entrevistado refiere:

... digamos que mi familia es elástica, a veces es de cinco y hasta siete personas. En otros momentos, la mayor parte del año somos dos personas, pero en un futuro vamos a ser tres personas permanentemen- te. Una familia muy dinámica, porque yo tengo dos separaciones, este es mi tercer matrimonio, tengo tres hijos y con mi actual esposa que llevamos siete años casados, estamos esperando un bebé. Entonces, cuando mis hijos están en receso académico del colegio convivimos todos al tiempo, en vacaciones, junio y julio, en diciembre, enero, en Semana Santa y nos reunimos todos en familia. Entonces es ahí cuando es la familia de las siete personas, y cuando estamos en las actividades ya normales somos dos personas, ella y yo, y tres con Sofía, que viene en camino. Por eso es como una dinámica elástica y en las épocas en que estamos como pareja disfrutamos de la vida en familia, igual cuando estamos todos salimos a pasear.

También es importante resaltar que la familia incide significativamente en la toma de decisiones económicas, puesto que esta misma se referencia al momento de tomarlas, debido a que afectarán directamente a todos sus miembros. Por tanto, la condición de estas personas a punto de pensionarse debe, a modo de sugerencia para continuar con el proyecto, verse avocada al fortalecimiento de las relaciones familiares que inmediatamente redundará en la satisfacción personal.

\section{Área personal}

Percepción de sí mismo y bienestar personal

La pregunta ¿Quién es usted? no fue fácil de responder para los funcionarios entrevistados, pues es una interrogante a la cual no se está acostumbrado. Por tanto, las respuestas evidencian que la constitución del yo se hace en relación con las cosas que hacen o la función que desempeñan socialmente.

En esta medida, se encuentran tres categorías principales desde donde las personas se configuran como sujetos: a) desde el cargo que desempeñan actualmente en la institución, b) la profesión o los estudios realizados que los avala como personas idóneas para desempeñar el cargo nombrado, y c) a través de la referencia de la estructura de la familia a la cual pertenece (padres, hijos, nietos). De manera aislada, algunos mencionaron ser "personas felices", pero no es una constante en las narrativas de estos funcionarios.

Las categorías encontradas dan cuenta de la relevancia que tiene para las personas su trabajo y el desa- 
rrollo profesional en su concepción de sí mismos y, por ende, en su desarrollo personal; es decir, es evidente la relación estrecha que existe entre su cotidianidad y el área laboral, pues excluyen otras áreas que también hacen parte de su vida diaria. Por otro lado, la familia también es una categoría constante en el discurso sobre el área personal, que denota la estructura, las edades de los integrantes, el tiempo de matrimonio, los estudios de los hijos y el lugar de la persona al interior de esa familia.

Se puede observar entonces, que existen tres figuras importantes para las personas, que se ven reflejadas a lo largo de las entrevistas y son: trabajo/profesiónfamilia-economía. Alrededor de estas tres figuras se estructuran los discursos de los entrevistados.

En cuanto al bienestar personal, las entrevistas permiten observar que en general los funcionarios consideran que ese concepto hace referencia a: "estar bien, sentirse feliz y en paz, compartir con la familia y tener equilibrio en todas las áreas de la vida". Aunque éste es el significado que le dan al concepto, algunas personas consideran que actualmente no tienen ese bienestar o que si lo tienen es sectorial. Por ejemplo, sólo perciben bienestar laboral o sólo bienestar en la familia; por lo tanto, esperan que una vez se hayan jubilado puedan obtener más tiempo y más tranquilidad.

Aquellas personas que refirieron ser y sentirse felices, consideran que en ese futuro cercano de la pensión no habrá cambios drásticos en su vida. Esta diferencia en la percepción del futuro, está directamente relacionada con la variabilidad y estructura de los proyectos de vida de cada persona, pues si ese plan de vida está claro y ha sido evaluado y planificado, no se espera a retirarse del trabajo para iniciar algo nuevo, sino que se vive en el aquí y el ahora.

\section{Historia de vida}

Se indagó con los participantes sobre aquellos recuerdos de situaciones significativas de su vida. Esto, con el ánimo de observar las áreas que tienen relevancia a lo largo de su historia de vida y que seguramente también lo son en el momento actual.

Se encontró que definitivamente el crecimiento de la familia, con el nacimiento de los hijos, es la situación más significativa para la mayoría de las personas en- trevistadas. Seguido a esta situación, los participantes refirieron recuerdos alrededor de su desarrollo profesional y laboral como relevantes a lo largo de su vida. Le siguen referencias sobre los estudios realizados y por último, los paseos y viajes familiares que han disfrutado. Pocas personas nombraron pérdidas de seres queridos como esposos, esposas y padres.

Se observa que nuevamente aparecen en las narrativas dos ejes de referencia: trabajo/profesión-familia.

\section{Proyecto de vida}

El proyecto de vida, aunque se conozca comúnmente de esta manera no es sólo un proyecto, sino que es una realidad que se vive día a día, es fundamental para todas las personas, pues está lleno de metas y motivaciones para lograrlas, es decir, atraviesa a las personas para conllevar comportamiento, acciones, actividades. En esto radica la importancia de poner especial atención en la estructura y el nivel de desarrollo del proyecto de vida de cada persona, pues se relaciona directamente con las motivaciones para seguir viviendo a pesar de no estar trabajando.

Entre los entrevistados se encontraron diferentes expectativas frente a las actividades a realizar después de la jubilación. Las más representativas tienen que ver con la creación de empresa/negocios, continuar trabajando, y descansar. En menor medida piensan en actividades como viajar, estudiar, vincularse a grupos eclesiásticos, docencia, comprar vivienda, etc. Por otro lado, aunque en pocos relatos, se encontró que hay personas que "no saben" o no han pensado en las actividades o el proyecto a seguir una vez que se retiren de laborar por motivo de pensión.

Se observa que hay variedad de actividades que las personas pueden y esperan realizar cuando ya no estén trabajando y esta variedad puede ser aprovechada para estructurar mucho más esos proyectos de vida particulares; sin embargo, llama la atención aquellas personas que no saben qué hacer una vez se jubilen, pues son las que más necesitan intervención para estructurar las metas a mediano y largo plazo y así prevenir posibles patologías derivadas de la desvinculación laboral, por ejemplo la depresión.

Asimismo, se deben tener en cuenta aquellas actividades que las personas, aunque lo tenían planeado, no han podido realizar aún, como hacer deporte, 
vincularse a actividades artísticas, dedicar tiempo a la familia, etcétera.

\section{Jubilación extemporánea}

Teniendo en cuenta que existen procesos de jubilación que pueden abarcar más tiempo que el esperado por los funcionarios, se indagó sobre su posible reacción, de manera que se pueda prever una posible intervención al respecto.

En esta área se evidenciaron básicamente tres tipos de respuesta: a) continuidad en el trabajo, b) frustración, y c) personas que se encuentran en esta situación actualmente.

Si se considera que varios de los prepensionados desearían continuar trabajando, la situación hipotética de sobrepasar los tiempos de pensión no les afecta, puesto que seguirían trabajando como hasta ahora. Sin embargo, existe otro grupo de personas que desean retirarse de laborar en el tiempo estipulado y, de no ser así, refieren que se sentirían decepcionados y frustrados porque sus planes se verían pospuestos.

Muy pocos relatos refieren estar en la situación de espera de pensión más tiempo del previsto, en especial, aluden al cambio del proceso a Colpensiones por el que está pasando la institución.

\section{Área comunitaria}

Esta área hace referencia a las relaciones sociales y las posibles redes de apoyo que pueden existir en la comunidad a la cual pertenecen los prepensionados de la sDs. Las preguntas de la entrevista se orientaron a develar las actividades que los funcionarios conocen se realizan en sus barrios y su disposición a participar en ellas, percepciones alrededor del bienestar comunitario y su posible aporte a éste, sus redes sociales y las relaciones interpersonales, y por último, los testimonios que conocen de personas ya pensionadas, en términos de ventajas y desventajas de la condición de jubilado.

\section{Actividades, participación y bienestar comunitario}

En las entrevistas se pudo observar que las personas en su mayoría, tienen conocimiento de las actividades que se realizan en su barrio y comunidad, son pocas las personas que no las reconocen. Las actividades que son más evidentes para ellos son las brigadas, las actividades culturales y la junta de acción comunal.
Se podría decir que la respuesta a la posible participación de actividades comunitarias se presenta dividida proporcionalmente entre las personas entrevistadas, pues cerca de la mitad no desea acceder o participar de ellas y la otra mitad sí está interesada en hacer parte de dichas actividades.

La percepción que presentan las personas del bienestar comunitario es que se relaciona con "vivir bien para que otros vivan bien", "bien común", "organización comunal”. Esta percepción es importante porque la mayoría quisiera vivir en un lugar donde se logre la convivencia, el respeto mutuo y la camadarería para poder integrarse más a la comunidad.

\section{Redes sociales y relaciones interpersonales}

La principal red social que tienen los funcionarios está conformada por sus compañeros de trabajo. Otras redes están integradas por amigos externos al trabajo, vecinos; unos pocos están vinculados a fundaciones o a iglesias con actividades de voluntariado.

En este apartado es importante destacar la poca referencia de los entrevistados sobre su participación en varias redes sociales o comunitarias, en lo cual es necesario intervenir, pues las redes son necesarias en la vida de las personas, ya que como tejido social ayudan a sostener a los sujetos que la conforman y en el ciclo vital de los prepensionados, estas redes facilitarían la realización y enriquecimiento del proyecto de vida.

\section{Testimonios cercanos}

En la entrevista se consideró importante indagar sobre el conocimiento de los funcionarios de testimonios de personas que ya están jubiladas, puesto que esto también aporta en la construcción de imaginarios sobre esta etapa.

Se pudo observar que la mayoría sí tiene personas cercanas que ya se han jubilado, tanto de la misma institución como de su familia. Entre las ventajas que se consideran durante esta nueva fase están la ampliación de tiempo para asuntos personales (salud, compras, etc.) y para dedicar a la familia; asimismo, perciben que las personas pensionadas tienen la oportunidad de viajar y son más felices.

Por otro lado, ven como desventaja del retiro laboral el distanciamiento de los amigos del trabajo, la disminución de ingresos económicos y las dificultades 
que a veces se presentan en las entidades para llevar a feliz término el proceso de jubilación.

\section{CONCLUSIONES}

En cuanto a los aspectos de salud, conviene concluir que, en términos generales, es adecuado suponer que los encuestados tienen un buen estado de salud general. Pueden encontrarse casos en los que no existe un reporte de alteración en alguna de las esferas del sujeto, como para generar alteración en el bienestar biopsicosocial.

En cuanto a la naturaleza de las enfermedades, conviene mencionar que todas ellas corresponden al deterioro de la función de algunos sistemas, producto de los cambios metabólicos asociados a las etapas del desarrollo en que se encuentran los encuestados, tales como fallos metabólicos (alteraciones en la regulación de la insulina, y las hormonas tiroideas), y otras cardiovasculares, secundarias a hábitos de vida y salud, situación que se relaciona estrechamente con la prevalencia e incidencia de este tipo de enfermedades en estos grupos de edad.

Los resultados muestran tipos de cuadro clínicos que normalmente no se asocian con la enfermedad profesional, por lo que conviene suponer que la condición de trabajo de la sDs no genera, preliminarmente, muestras de afectación en la ocupación del empleado, incluso posterior a un proceso extendido y continuo de actividad laboral, tal como puede verse en los años de antigüedad descritos por los encuestados.

Antes de cumplir los requisitos legales y de concienciar que se aproxima la etapa de jubilación, es importante sensibilizar no sólo al individuo, sino también a su entorno familiar y social, para prepararlos a lo que supone un nuevo momento en su ciclo vital.

Aquellas personas con una rutina satisfactoria son conscientes de sus capacidades y limitaciones, conocen lo que se espera de ellos y tienen asumido su propio rol o papel. Para algunas personas, el papel del jubilado es irrelevante, debido a que vuelven al trabajo o debido a una enfermedad o una incapacidad.

Pese a que se tiene una cultura del ahorro, los entrevistados evidencian no haber proyectado una situación que los llegase a dejar sin solvencia económica. Este aspecto incide de manera importante en la vida del pensionado, ya que sus aspiraciones se basan en tener una estabilidad económica en su vejez, sin depender ni ser una carga para nadie, es la satisfacción y la recompensa a todo el trabajo que realizó por años.

La relación estrecha entre la configuración del yo y el área laboral se debe tener en cuenta en la preparación para la jubilación, pues al presentarse un cese de las actividades laborales puede entrar en crisis el yo (la persona), por lo cual podría previamente construirse otras relaciones entre la percepción personal y otras áreas de la vida (comunitaria, familia, etcétera).

La intervención en el área personal debe tener en cuenta el nivel de estructuración y de desarrollo del proyecto de vida de cada prepensionado, de manera que la asesoría al respecto sea pertinente como acompañamiento para direccionarlo, re-direccionarlo o incluso generarlo con la persona participante del programa. En el área comunitaria, los prepensionados evidencian poca adhesión a redes sociales o redes de apoyo. Es importante intervenir en esta área, ya que las redes son un posible y efectivo soporte para el jubilado que, además, está en la etapa de adultez mayor del ciclo vital.

Por último, es evidente que las personas que están próximas a retirarse de la sDs por motivo de pensión aprecian que la institución esté diseñando y trabajando en la creación del Programa de prepensionados, pues lo perciben como la oportunidad para prepararse para una nueva etapa y así propender por una adecuada calidad de vida. • 


\section{REFERENCIAS}

Cuartas, M. E. (1999). Preparación para la jubilación $y$ el papel de la familia en este proceso. Colombia: Pontificia Universidad Javeriana.

De Freitas, L. \& Vaughan, G. (2008). Ganhos e perdas: atitudes dos executivos brasileiros e neozelandeses frente à aposentadoria. Psicologia em Estudo, 2, 207-216.

Eguiluz, L. (2007). Dinámica de la familia un enfoque psicológico académico. Recuperado de http://books. google.com.co/books?id=qY2ngOIP88gC\&hl=es\&s ource $=$ gbs_navlinks_s

Fernández, J. Crego, A. \& Alcover, C. (2008). Relaciones entre factores sociodemográficos, motivación hacia el retiro temprano y satisfacción en la vida poslaboral: análisis exploratorio en una muestra de prejubi- lados españoles. Revista de Psicología del Trabajo y de las Organizaciones, 24(3), 433-436.

Giró, M. (2009). Envejecimiento, tiempo libre y gestión del ocio. España: Universidad de La Rioja.

Placeres, J., Lázaro, R. \& Delgado, I. (2011). The family and the elder adult. Revista Matanzas, 14, 472-483. Recuperado de www.revmatanzas.sld.cu/revista\%20 medica/ano\%202011/vol4\%202011/tema10.htm

Potocnik, K., Tordera, N. \& Peiró, J.M. (2008). Ajuste al retiro laboral en función del tipo de retiro y su voluntariedad desde una perspectiva de género. Revista de Psicología del Trabajo y de las Organizaciones, 24(3), 347-364.

Secretaría Distrital de Salud. (2013). Recuperado de www.saludcapital.gov.co/Paginas/QuienesSomos. aspx en el apartado de Misión Institucional. 